weiteren Faktoren konnte keinen Unterschied der brustkrebsassoziierten Sterblichkeit bezogen auf brusterhaltende Therapien mit Bestrahlung versus Mastektomie zeigen. Damit ist das Outcome von DCIS vergleichbar mit kleinen invasiven Karzinomen. Bei beiden sind Tumorgröße und Grading Prädiktoren für Mortalität, vor allem bei HR-positivem Befund in den ersten zehn Beobachtungsjahren. Ebenfalls in beiden Situationen ist das Risiko unter dem 40. Lebensjahr und bei bestimmen Ethnizitäten höher, und die Mortalität steigt nach Brustrezidiv (invasiv). Während HR-positive DCIS in den ersten zehn Jahren ein geringeres Mortalitätsrisiko hatten als HR-negative, glich sich dies in den 20-Jahres-Daten aus. G3 war nicht assoziiert mit häufigerem Auftreten ipsilateraler invasiver Karzinome, aber mit einer erhöhten brustkrebsassoziierten Gesamtsterblichkeit (Faktor 1,8).
Einige DCIS besitzen trotz lokaler Kontrolle das Potenzial, analog kleinen invasiven Karzinomen, Fernmetastasen zu bilden und die Brustkrebssterblichkeit ohne invasive Diagnose zu erhöhen. Das daraus resultierende (wenn auch geringe) Mortalitätsrisiko nach DCIS muss berücksichtigt, Einflussfaktoren (etwa Resektionsränder, systemische Therapien) müssen weiter erforscht werden. Voraussetzung für die prognostische Entscheidung ist vor allem die gründliche pathologische Aufarbeitung zur Differenzierung zwischen DCIS und invasivem Karzinom.

Auch wenn Follow-up-Daten über 20 Jahre hinaus und höhere Fallzahlen sowie eine zweite zentrale Pathologiebefundung wünschenswert gewesen wären, so zeigt die Studie doch, dass die sorgfältige interdisziplinäre Zusammenarbeit zwischen Diagnostikern, Operateuren, Strahlen- und
Systemtherapeuten auch beim DCIS analog den aktuellen Leitlinien für manche Betroffene eine lebensentscheidende Rolle spielen kann. Auch die Nachsorge sollte bildgebend wie allgemein mit der gleichen Sorgfalt wie beim invasiven Karzinom durchgeführt werden. Zudem muss die Forschung hinsichtlich Grundlagen des DCIS und der gegebenenfalls individualisierten lokalen wie systemischen Therapien besonders aufgrund steigender DCIS-Detektionsraten im Screening weiter unterstützt werden.

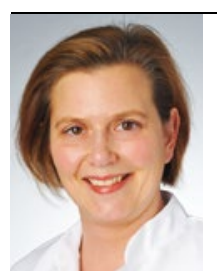

Dr. med. Rachel Würstlein Geschäftsführende Oberärztin, Brustzentrum der Universität München

\title{
Geburtsverlauf: das Köpfchen entscheidet, nicht das Gewicht
}

\section{Ein Kopfumfang jenseits der 95. Perzentile ist offenbar häufiger mit Geburts- komplikationen verknüpft als ein vergleichbar hohes Geburtsgewicht.}

n einer auf Krankenakten gestützten Studie wurden ca. 25.000 Geburten, darunter mehr als 6.000 Erstgeburten, analysiert, um Einflussgrößen zu identifizieren, die das Risiko für Geburtskomplikatio-

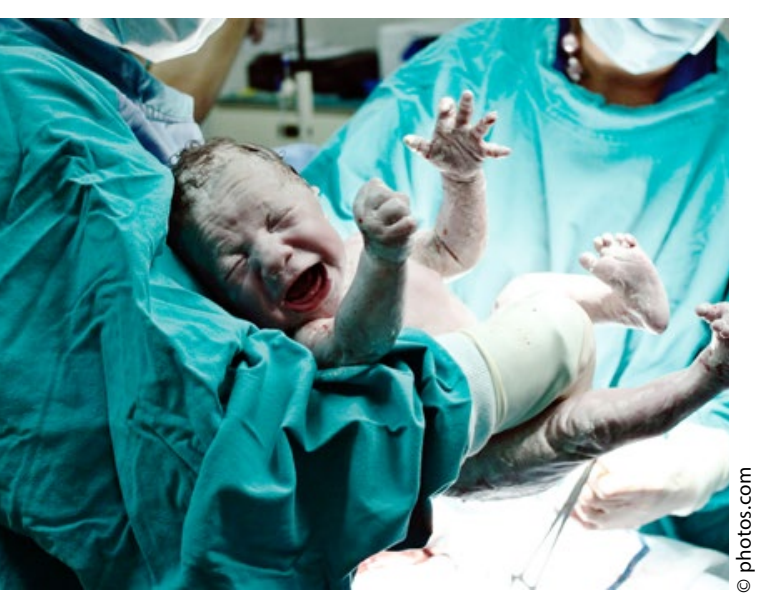

Kinder mit großem Kopfumfang müssen häufiger durch einen ungeplanten Kaiserschnitt zur Welt gebracht werden nen, ungeplante Kaiserschnitte und instrumentelle Geburten erhöhen. Üblicherweise liegt hier der Fokus auf einem hohen Geburtsgewicht („big baby“). Lipschuetz et al. vermuteten aber, dass der Kopfumfang den Geburtsverlauf stärker beeinflusst als das Gewicht.

Lag der (postnatal gemessene) Kopfumfang des Kindes jenseits der 95. Perzentile, wurde häufiger ein nicht geplanter Kaiserschnitt oder eine instrumentelle Intervention nötig. Bei hohem Geburtsgewicht hingegen ist dies nur dann der Fall, wenn auch der Kopf sehr groß ist. Beides fällt keineswegs regelhaft in eins: nur 24\% der "big babies" hatten auch einen großen Kopfumfang. Der Anteil solcher Babys an der Gesamtkohorte betrug rund 2\% und knapp $6 \%$ hatten große Köpfe.

$62 \%$ der Kinder mit großem Köpf wurden vaginal geboren, $16 \%$ mittels Kaiserschnitt und $11 \%$ unterstützend durch Instrumente. Bei kleinerem Kopf betrugen die Quoten 78\%, 8\% und 7\%. (zu 100\% fehlende Anteile entfielen auf elektive Sec- tiones.) Das Chancenverhältnis, mit großem im Vergleich zu einem kleinen Kopf auf eine ungeplante Sectio zuzusteuern, betrug 2,6, jenes für eine instrumentelle Entbindung 2,1. Dagegen wurden Kinder mit hohem Geburtsgewicht zu $80 \%$ vaginal entbunden. Die Raten für ungeplanten Kaiserschnitt bzw. Instrumentengeburt betrugen $10 \%$ bzw. 3\%. Gegenüber normalgewichtigen Kindern war das Chancenverhältnis für komplizierte Entbindungen kaum erhöht bzw. sogar erniedrigt. Das hohe Gewicht hatte bei normalem Kopfumfang keinen Einfluss auf die Komplikationsrate. Nur wenn der Kopf ebenfalls groß war, stieg das Chancenverhältnis für einen Kaiserschnitt auf 1,9. Die weitere Analyse ergab, dass Kaiserschnitte bei Kindern mit großem Kopf häufiger durch ausbleibenden Geburtsfortschritt bedingt waren. Bei Kindern mit geringerem Kopfumfang gaben eher fetale Komplikationen den Anlass zur Sectio.

Die Autoren fordern nun prospektive Studien, um zu klären, ob sich mit pränatalen Messungen des kindlichen Kopfumfangs der Geburtsverlauf vorhersagen lasse.

Dr. Robert Bublak

Lipschuetz $M$ et al. A large head circumference is more strongly associated with unplanned cesarean or instrumental delivery and neonatal complications than a high birth weight. Am J Obstet Gynecol 2015; doi: 10.1016/j.ajog.2015.07.045 